\title{
MODELOS SINTÉTICOS DE APRENDIZAGEM PARA APLICAÇÃO DE INJEÇÃO PELAS VIAS INTRAMUSCULAR E SUBCUTÂNEA EM CÃES
}

(Synthetic models for learning intramuscular and subcutaneous techniques of injections administration in dogs)

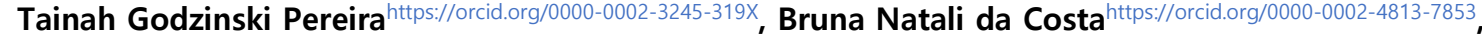

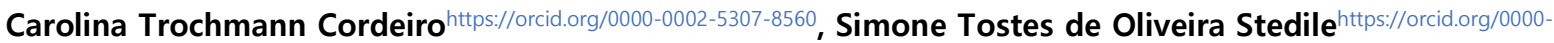
0002-8218-379x
}

Universidade Federal do Paraná, Departamento de Medicina Veterinária, Curitiba, PR, Brasil.

*Autor para correspondência: tostesimone@gmail.com

Editora: Julia Arantes Galvão

RESUMO - Na Medicina Veterinária, a aplicação de injeções é uma das habilidades clínicas que pode causar ansiedade nos estudantes, interferindo na aprendizagem. O objetivo do presente estudo foi desenvolver e validar modelos sintéticos de aprendizagem para aplicação de injeção pelas vias intramuscular e subcutânea em cães. A metodologia incluiu a confecção de três modelos sintéticos, sendo um para administração de injeção intramuscular no membro pélvico, outro para injeção intramuscular nos músculos lombodorsais e um terceiro para injeção subcutânea. Foram utilizadas borrachas de silicone de diferentes densidades para a obtenção das consistências desejadas. Os modelos foram acoplados em manequins de pelúcia e passaram por avaliação de médicos veterinários experientes. Após ajustes sugeridos, os modelos foram utilizados em aula por 69 estudantes da disciplina de Semiologia Geral Veterinária. No final da aula, os estudantes responderam a um questionário, para opinar sobre o aprendizado com os modelos, que contava com questões em escala Likert de 5 pontos e questões abertas. A maioria dos estudantes $(98,4 \%)$ concordou ou concordou fortemente quanto à contribuição dos métodos utilizados para seu aprendizado e 92,6\% disseram se sentir mais confiantes para aplicação de injeção no animal vivo. Após aula com o modelo sintético, 20 estudantes tiveram a oportunidade de aplicar injeções pela via subcutânea em cães vivos, sendo que 100\% dos estudantes concordaram ou concordaram fortemente que o modelo sintético auxiliou na realização da técnica de injeção no animal vivo. Os modelos se mostraram uma ferramenta eficiente para o aprendizado de aplicação de injeção pelas vias intramuscular e subcutânea em cães.

Palavras-chave: educação; ensino humanitário; habilidades clínicas; métodos substitutivos; substituição. 
ABSTRACT - Administration of injections is one of the clinical skills that can cause anxiety in veterinary students, interfering in the learning process. The aim of this study was to develop and validate synthetic learning models for the administration of intramuscular and subcutaneous injections in dogs. The methodology included the development of three synthetic models: one for administration of intramuscular injection in the pelvic limb, another for intramuscular injection in the lumbodorsal muscles and a third for subcutaneous injection. Silicone rubbers of different densities were used to obtain the desired consistencies. The models were attached to stuffed dog toys and were evaluated by experienced veterinarians. After suggested adjustments, the models were used by 69 students in the discipline of Veterinary Semiology. At the end of the class, the students completed a questionnaire on their experience of learning with the models. Questions included those with answers on a 5-point Likert scale and open questions. Most students (98.4\%) strongly agreed or agreed that the method was useful for their learning and $92.6 \%$ said they felt more confident about giving an injection to a live animal. After the class with the synthetic model, 20 students had the opportunity to give subcutaneous injections to live dogs. All these students agreed or strongly agreed that experience of the model had helped them perform the injection technique in live animals. The models proved to be an efficient tool for teaching administration of injection by intramuscular and subcutaneous routes in dogs.

Keywords - clinical skills, humane teaching; medical education; replacement; alternative methods.

\section{INTRODUÇÃO}

Na Medicina Veterinária, para que o objetivo da terapêutica seja alcançado, é necessário que o médico veterinário e toda sua equipe sejam devidamente treinados quanto às técnicas para aplicação de injeções (CROW \& WALSHAW, 2000). O responsável pelo procedimento deve ter compromisso ético e conhecimentos técnicos sobre vias de administração, fármacos e suas ações, doses e efeitos colaterais. A fim de diminuir a incidência de erros iatrogênicos, são recomendadas capacitações e educação continuada aos profissionais da área (TELLES FILHO \& CASSIANI, 2004). Em um primeiro momento pode-se pensar que aulas com pacientes vivos seriam ideais para o aprendizado de tais técnicas, entretanto, há fatores que refutam esta abordagem tradicional de ensino. Há indícios de que aulas com esta característica podem gerar tensão nos estudantes (NAYLOR et al,. 2009), desconforto e sentimentos negativos como ansiedade, angústia, pena, tristeza, nervosismo e medo por parte dos estudantes (DINIZ et al., 2006; LANGEBAEK et al., 2012; TRÉZ, 2015). Portanto, tem-se buscado métodos humanitários de substituição, proporcionando ensinamentos clínicos aos estudantes de Medicina 
Veterinária de forma ética, como o uso de modelos e simuladores (SMEAK, 2003). Inicialmente, o custo de investimento para a fabricação e desenvolvimento de tais métodos pode parecer alto, mas com o tempo o custo-benefício é percebido, uma vez que os modelos podem ser reutilizados sem que haja gastos adicionais, que estariam presentes com o uso de animais vivos (GREIF \& TRÉZ, 2000; MAGALHÃES \& ORTÊNCIO FILHO, 2006). Muitos estudos já mostraram ser possível implementar esta abordagem metodológica, mantendo a qualidade de ensino (DINIZ, 2006; KNIGHT, 2007; CARDOSO \& LANGE, 2015), além da possibilidade de esta acarretar maior assimilação do conteúdo prático por parte dos estudantes (GREIF \& TRÉZ, 2000; KNIGHT, 2007; CANOVA, 2015). Apesar da importância dos métodos substitutivos no ensino, os autores não encontraram, na literatura ou comercialmente, modelos sintéticos criados para injeção pelas vias intramuscular e/ou subcutânea em cães, que mostrem a localização anatômica como referência dos pontos para injeção no animal. O objetivo deste trabalho foi desenvolver e validar modelos sintéticos para treinamento de injeções intramuscular e subcutânea em cães, proporcionando consistência e textura dos músculos e tecido subcutâneo semelhantes ao de um animal real.

\section{MATERIAIS E MÉTODOS}

\section{Confecção dos modelos}

Foram desenvolvidos três modelos sintéticos distintos, de baixo custo, para o treinamento de aplicação de injeções em cães: modelo para injeção intramuscular nos músculos da região femoral, modelo de injeção intramuscular nos músculos lombodorsais e modelo de injeção subcutânea. Previamente à confecção dos modelos, grupos musculares da região femoral e lombodorsal de cães vivos foram palpados, com maior atenção direcionada às densidades que apresentavam, bem como sua topografia em relação a outras estruturas, como protuberâncias ósseas da região. Foram avaliadas também a consistência e flexibilidade do tecido subcutâneo da região dorsal.

Para a confecção do modelo de injeção intramuscular nos músculos da região femoral, balões de látex foram preenchidos com borracha de silicone de média flexibilidade fracionada em pequenos fragmentos, a fim de simular a musculatura da área usada para injeção intramuscular. Para representar a parte óssea, foram utilizados fragmentos de arame envolvidos com polimetilmetacrilato. O modelo foi feito em duplicata e inserido nos membros pélvicos de um boneco de pelúcia, para melhor simular a região anatômica (Figura 1). 


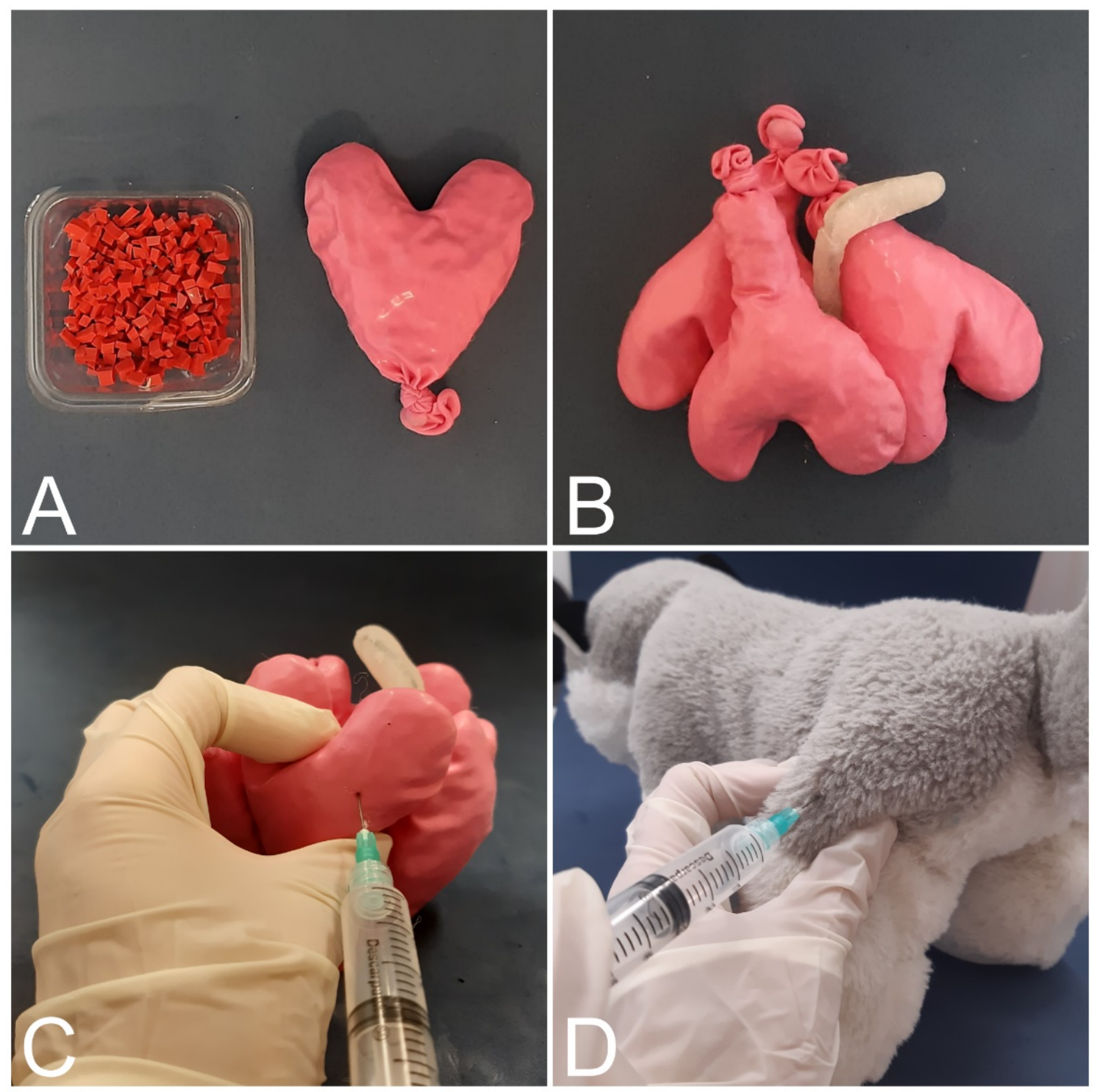

Figura 1 - Confecção do modelo para injeção intramuscular na região femoral de cão ABorracha de silicone fracionada em pequenos fragmentos (esquerda) e balão de látex preenchido por silicone (direita). B- Conjunto representativo dos grupos musculares e do fêmur. C- Simulação óssea e muscular da região femoral, mostrando um dos possíveis locais de injeção na coxa. D- Modelo já pronto, revestido com o boneco de pelúcia, mostrando o mesmo local de aplicação da injeção de C. Para a simulação de cada membro pélvico foram necessários quatro balões de látex preenchidos com silicone fracionado. Para melhor assentamento dos balões, foi utilizada uma garrafa no interior do boneco, para dar forma ao corpo e impedir que os balões de látex se deslocassem dos membros.

Para a confecção do modelo de injeção intramuscular nos músculos lombodorsais, foram usados dois fragmentos de câmara de ar para bicicleta com diâmetro de dois centímetros, para simular tais músculos. As câmaras foram preenchidas com borracha de silicone de média flexibilidade, fracionada em pequenos fragmentos, e suas extremidades 
foram fechadas com elástico de látex. Foi adicionada uma peça produzida a partir de arame de alumínio e massa de biscuit, modelada para representar a crista ilíaca e coluna vertebral do animal vivo, ressaltando os processos espinhosos. O modelo foi inserido em um boneco de pelúcia modificado para este fim (Figura 2).
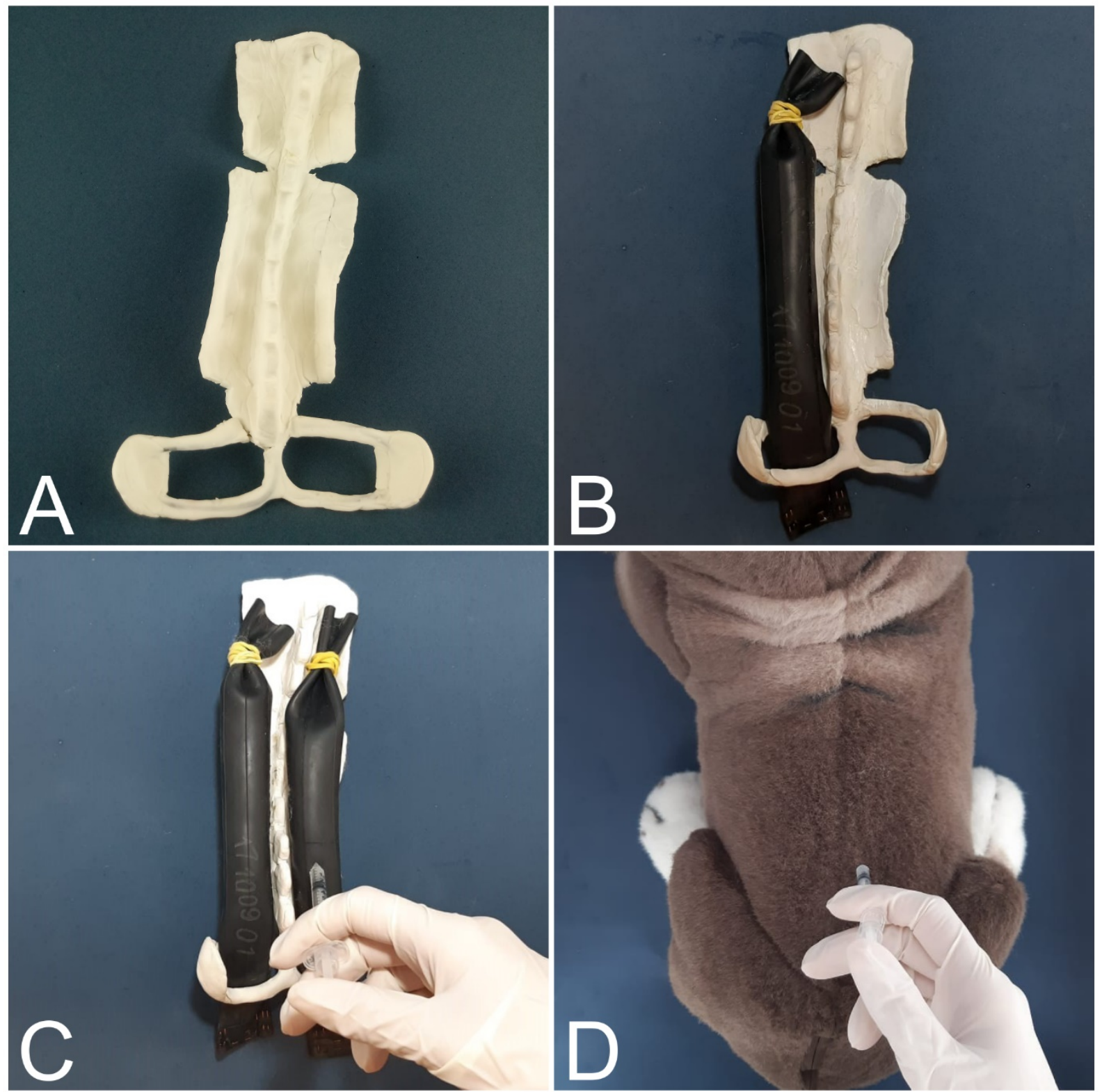

Figura 2 - Confecção do modelo para injeção intramuscular nos músculos lombodorsais de cão. A- Crista ilíaca e coluna vertebral moldada a partir de massa de biscuit, simulando processos espinhosos. B- Simulação da musculatura lombodorsal unilateral, crista ilíaca e coluna toracolombar, permitindo a palpação destas estruturas como pontos de referência para injeção. C- Simulação da musculatura bilateral, mostrando o local de injeção. D- Modelo já pronto, revestido com o boneco de pelúcia, mostrando o local de aplicação das injeções. Para a confecção de cada grupo muscular foi utilizado silicone fracionado em pequenos fragmentos inseridos em borracha de câmara de ar. O osso coxal (ílio) foi ajustado de acordo com a posição do boneco de pelúcia, a fim de melhor representação anatômica, como pode ser observado na figura B. 
Para o desenvolvimento do modelo de injeção subcutânea, foram simuladas as camadas muscular e subcutânea. A camada muscular foi confeccionada com borracha de silicone de média flexibilidade e a camada subcutânea com elastômero de silicone de baixa viscosidade 00-30 sobre tecido fenestrado. Cada camada foi confeccionada separadamente e, após a cura dos materiais, tiveram suas bordas unidas com silicone acético. Para que houvesse possibilidade de deslizamento entre as estruturas, foi adicionado óleo mineral no espaço livre entre a camada muscular e a subcutânea. Esse aspecto de movimento foi de suma importância para a execução da técnica de injeção. Em seguida, ambas as camadas foram colocadas em volta de uma garrafa de vidro e fixadas nas extremidades da mesma com elástico, para que simulassem o formato do corpo do animal. O conjunto foi inserido em um boneco de pelúcia, de forma que toda a região dorsal e lateral do corpo tivessem a possibilidade da aplicação de injeções subcutâneas (Figura 3).

Para a injeção em todos os locais, foi utilizado álcool como o líquido a ser aplicado, para que os modelos não fossem danificados e pudessem ser reutilizados. $O$ álcool foi acondicionado em frasco ampola, em substituição a diluentes de medicamentos. Desta forma, o álcool era puxado para a seringa sem que seu odor fosse evidente, simulando o uso de fármacos em geral. A escolha do álcool se deu por experiências prévias com a utilização de solução fisiológica ou água e posterior contaminação fúngica ou bacteriana dos modelos. A cada dia de uso do modelo de injeção subcutânea se fazia necessário a adição de óleo mineral no espaço subcutâneo, em volume aproximado de $5 \mathrm{~mL}$, para que uma camada deslizasse sobre a outra.

\section{Validação de conteúdo}

Os modelos sintéticos foram submetidos à validação de conteúdo por sete médicos veterinários experientes, residentes de diferentes áreas, por meio de um questionário anônimo dividido em duas partes. A primeira parte era composta por questões com respostas graduadas em escala Likert contendo cinco alternativas, variando de discordo fortemente a concordo fortemente, conforme o grau de concordância dos profissionais com a afirmativa. Essas questões abordavam os aspectos estruturais dos modelos, a proximidade com o animal vivo em consistência, elasticidade, tamanho e textura durante a manipulação e aplicação de medicações. A segunda parte abordava, por meio de questões abertas, a aceitação dos modelos, nível de eficiência para treinamento das técnicas e se seriam viáveis para aplicação no ensino. 

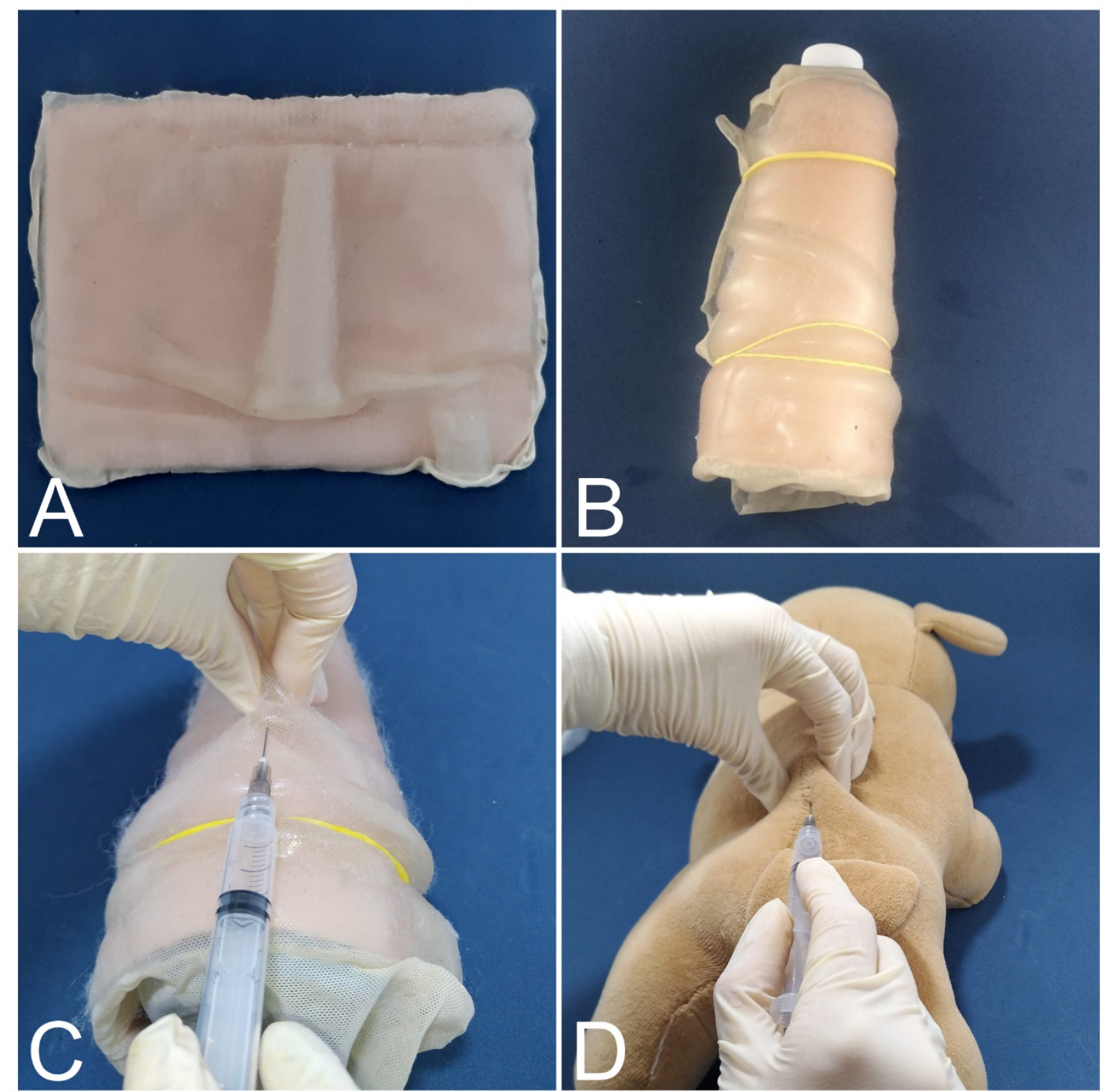

Figura 3 - Confecção do modelo para injeção subcutânea. A- Camada subcutânea simulada por silicone e tecido fenestrado sobre a camada muscular (silicone rosa). Entre as duas camadas foi adicionado óleo mineral. A elevação central foi criada propositalmente para mostrar a formação da prega cutânea para aplicação de injeção durante a palpação do tecido subcutâneo. B- Garrafa envolta pelas camadas muscular e subcutânea, fixadas nas extremidades. C- Simulação das camadas subcutânea e muscular do animal. Observar a elasticidade da camada externa do silicone, permitindo que seja simulada a prega cutânea para inserção da agulha e correta aplicação da injeção. DModelo já pronto, revestido com o boneco de pelúcia, mostrando o local de aplicação das injeções.

\section{Validação de construto}

Após a realização das modificações sugeridas pelos médicos veterinários, os modelos foram utilizados na aula prática da disciplina de Semiologia Geral Veterinária. 
Participaram da aula 69 estudantes, sendo que 43 destes cursaram a disciplina no primeiro semestre do ano letivo, e 26 no semestre seguinte. Os estudantes foram divididos em grupos contendo em média cinco participantes, visando assim melhor orientação individual e melhor aproveitamento por parte dos mesmos. Todos os grupos foram acompanhados pelos mesmos (três) instrutores, e o tempo da aula de cada grupo foi de aproximadamente 20 minutos. Primeiramente, o grupo recebeu explicações sobre os locais de injeções, mostrados no livro de Semiotécnica de Pequenos Animais (TAYLOR, 2001). Então, os estudantes utilizaram cães saudáveis para palpar os locais de injeções. Os cães participaram apenas desta etapa (de palpação) e, portanto, não receberam nenhuma injeção. Na sequência, os estudantes fizeram o treinamento de injeção pelas vias intramuscular e subcutânea nos modelos sintéticos (Figura 4).

Cada estudante aplicou pelo menos uma injeção em cada local dos modelos sintéticos (membro pélvico, musculatura lombodorsal e aplicação subcutânea no dorso), sendo permitida a repetição caso o estudante assim o quisesse. Durante a aplicação, os instrutores orientavam e tiravam dúvidas para que o procedimento fosse realizado corretamente. Após o treinamento, os estudantes responderam um questionário anônimo, para opinar sobre a contribuição dos métodos apresentados para seu aprendizado. O questionário seguiu um padrão similar ao dos médicos veterinários experientes, com respostas graduadas em escala Likert e questões abertas.

\section{Validação de concorrência}

Após aula prática com os modelos, os estudantes que cursaram a matéria no segundo semestre tiveram a oportunidade de aplicação de injeção pela via subcutânea em cães vivos, dois meses após a aula com os modelos. Ao todo, 20 estudantes compareceram à essa aula prática, realizada em acordo com um canil, para vacinação anual de 16 cães da raça Beagle. Foram aplicadas duas vacinas em cada cão, sendo uma antirrábica e uma polivalente, totalizando 32 aplicações. Cada estudante pôde aplicar pelo menos uma injeção, sendo que alguns estudantes puderam fazer mais de uma aplicação. Os estudantes responderam a um segundo questionário com questões comparando as duas experiências, com modelos e animais vivos, para avaliar se o modelo havia contribuído positivamente em seu treinamento prévio. 

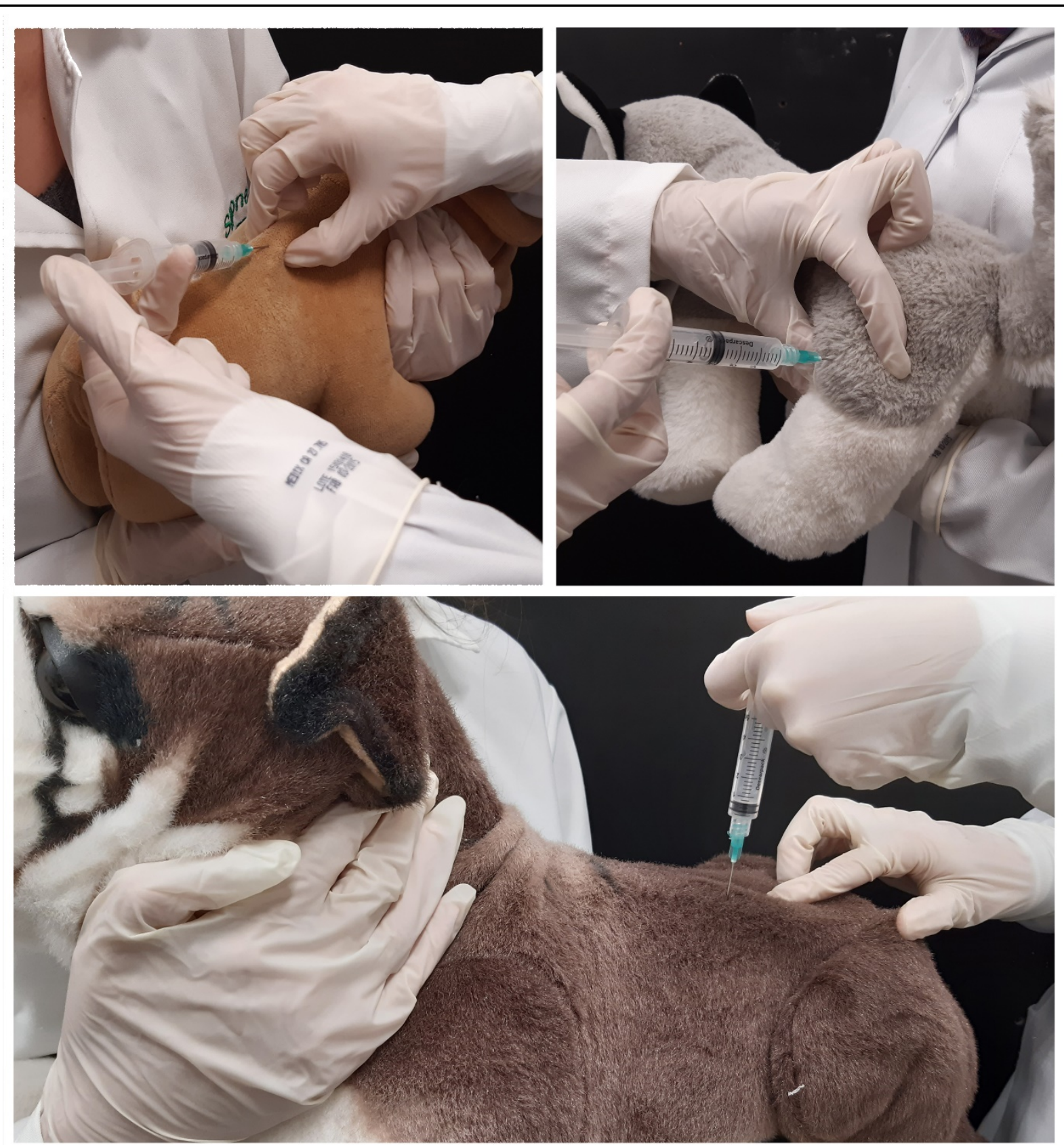

Figura 4 - Estudantes utilizando os modelos para treinamento de aplicação de injeção pelas vias intramuscular e subcutânea, durante a aula de Semiologia Geral Veterinária. Observe que os estudantes puderam treinar a contenção dos animais e puderam optar pela utilização ou não de luvas de procedimento.

\section{RESULTADOS E DISCUSSÃO}

Na literatura não foram encontrados modelos sintéticos para injeção intramuscular e/ou subcutânea em cães, sendo este o primeiro trabalho a desenvolver modelos sintéticos para este objetivo. Os modelos confeccionados atingiram os objetivos desejados, apresentando espessuras e textura semelhantes às dos animais reais, conforme observado na validação de conteúdo pelos profissionais. Em resposta às 
questões objetivas, todos os médicos veterinários experientes concordaram quanto ao tamanho dos modelos sintéticos utilizados serem adequados para representarem pacientes caninos, bem como as referências anatômicas incluídas nos modelos (Tabela 1).

Tabela 1 - Resposta dos médicos veterinários experientes $(n=7)$ quanto às características do modelo, em comparação ao animal real, e sua utilidade no ensino de aplicação de injeção intramuscular e subcutânea.

\begin{tabular}{|c|c|c|c|c|c|}
\hline $\begin{array}{l}\text { Em relação aos modelos apresentados para } \\
\text { aplicação de injeções em cães: }\end{array}$ & $\mathrm{DF}^{1}$ & $\mathbf{D}^{2}$ & $\mathbf{N}^{3}$ & $\mathrm{C}^{4}$ & $\mathrm{CF}^{5}$ \\
\hline $\begin{array}{l}\text { Os tamanhos dos modelos foram apropriados } \\
\text { para a manipulação }\end{array}$ & 0 & 0 & 0 & $\begin{array}{c}1 \\
(14,3 \%)\end{array}$ & $\begin{array}{c}6 \\
(85,7 \%)\end{array}$ \\
\hline $\begin{array}{l}\text { Um número adequado de estruturas de } \\
\text { referências anatômicas estava presente nos três } \\
\text { modelos }\end{array}$ & 0 & 0 & $\begin{array}{c}1 \\
(14,3 \%)\end{array}$ & $\begin{array}{c}1 \\
(14,3 \%)\end{array}$ & $\begin{array}{c}5 \\
(71,4 \%)\end{array}$ \\
\hline Os modelos foram fáceis de usar & 0 & 0 & 0 & $\begin{array}{c}1 \\
(14,3 \%)\end{array}$ & $\begin{array}{c}6 \\
(85,7 \%)\end{array}$ \\
\hline $\begin{array}{l}\text { A sensação à palpação foi realista para o modelo } \\
\text { de injeção intramuscular no membro pélvico }\end{array}$ & 0 & 0 & $\begin{array}{c}1 \\
(14,3 \%)\end{array}$ & $\begin{array}{c}2 \\
(28,6 \%)\end{array}$ & $\begin{array}{c}4 \\
(57,1 \%)\end{array}$ \\
\hline $\begin{array}{l}\text { A sensação à palpação foi realista para o modelo } \\
\text { de injeção intramuscular lombodorsal }\end{array}$ & 0 & 0 & $\begin{array}{c}1 \\
(14,3 \%)\end{array}$ & $\begin{array}{c}1 \\
(14,3 \%)\end{array}$ & $\begin{array}{c}5 \\
(71,4 \%)\end{array}$ \\
\hline $\begin{array}{l}\text { A sensação à palpação foi realista para o modelo } \\
\text { de injeção subcutânea }\end{array}$ & 0 & 0 & 0 & $\begin{array}{c}1 \\
(14,3 \%)\end{array}$ & $\begin{array}{c}6 \\
(85,7 \%)\end{array}$ \\
\hline $\begin{array}{l}\text { A sensação à aplicação de injeção foi realista para } \\
\text { o modelo de injeção intramuscular }\end{array}$ & 0 & 0 & $\begin{array}{c}1 \\
(14,3 \%)\end{array}$ & $\begin{array}{c}4 \\
(57,1 \%)\end{array}$ & $\begin{array}{c}2 \\
(28,6 \%)\end{array}$ \\
\hline $\begin{array}{l}\text { A sensação à aplicação de injeção foi realista para } \\
\text { o modelo de injeção subcutânea }\end{array}$ & 0 & 0 & 0 & $\begin{array}{c}1 \\
(14,3 \%)\end{array}$ & $\begin{array}{c}6 \\
(85,7 \%)\end{array}$ \\
\hline $\begin{array}{l}\text { O modelo seria adequado para ensinar aplicação } \\
\text { de injeção intramuscular nos músculos da região } \\
\text { femoral aos estudantes }\end{array}$ & 0 & 0 & $\begin{array}{c}1 \\
(14,3 \%)\end{array}$ & 0 & $\begin{array}{c}6 \\
(85,7 \%)\end{array}$ \\
\hline $\begin{array}{l}\text { O modelo seria adequado para ensinar aplicação } \\
\text { de injeção intramuscular nos músculos } \\
\text { lombodorsais aos estudantes }\end{array}$ & 0 & 0 & 0 & $\begin{array}{c}1 \\
(14,3 \%)\end{array}$ & $\begin{array}{c}6 \\
(85,7 \%)\end{array}$ \\
\hline $\begin{array}{l}\text { O modelo seria adequado para ensinar aplicação } \\
\text { de injeção subcutânea aos estudantes }\end{array}$ & 0 & 0 & 0 & $\begin{array}{c}1 \\
(14,3 \%) \\
\end{array}$ & $\begin{array}{c}6 \\
(85,7 \%) \\
\end{array}$ \\
\hline
\end{tabular}

${ }^{1} \mathrm{DF}$ : Discordo fortemente, ${ }^{2} \mathrm{D}$ : Discordo, ${ }^{3} \mathrm{~N}$ : Neutro, ${ }^{4} \mathrm{C}$ : Concordo, ${ }^{5} \mathrm{CF}$ : Concordo fortemente

Nas questões abertas, como pontos fortes, $6(85,7 \%)$ médicos veterinários citaram a semelhança dos modelos com o animal real, em relação às características físicas de introdução da agulha e aplicação de medicamentos, bem como a oportunidade de estudantes destreinados aprenderem sem causar dor ao animal real. De acordo com Greif \& Tréz (2000), os métodos substitutivos possibilitam melhor aprendizado por parte dos estudantes inexperientes, uma vez que permitem que cada indivíduo aprenda de acordo 
com seu ritmo, podendo utilizar repetição de treino, quando necessário. Um dos pontos fracos apontados foi a ausência de vácuo ao se puxar o êmbolo da seringa, citado por 3 $(42,9 \%)$ médicos veterinários. Neste quesito o modelo apresenta reduzida fidelidade à resposta do êmbolo da seringa, entretanto, este detalhe não impediu que o objetivo geral do trabalho fosse atingido. Nesta etapa do aprendizado, é importante que o estudante consiga identificar os locais de injeção corretamente, bem como aprenda a manipular seringas. Desta forma, os modelos não têm o objetivo de substituir por completo o treinamento com o uso de animais e sim estimular o desenvolvimento das habilidades básicas, visando reduzir a ocorrência de erros iatrogênicos. Nesta fase de treinamento com seringas, é possível observar em muitas aulas práticas, a dificuldade dos estudantes para aspirar medicações ou segurar a seringa adequadamente, sendo comum a tendência dos estudantes inexperientes de segurar a seringa da mesma forma que empunham uma caneta. Tais deficiências são supridas por meio da experiência e treinamento com os modelos. Outro ponto fraco citado foi a falta de reação do animal, mencionado por 1 (14,3\%) profissional; entretanto, um estudo demonstrou que a maioria dos estudantes têm emoções negativas durante aula prática com animais vivos, sendo que estas interferem na concentração e consequentemente no processo de aprendizagem (LANGEBAEK et al., 2012). Desta forma, apesar de aqui apontado pelo médico veterinário como ponto negativo, poderia ser na verdade um ponto positivo nesta etapa do ensino.

Nos questionários, 68 (98,6\%) estudantes concordaram ou concordaram fortemente que os modelos contribuíram para seu aprendizado e 64 (92,8\%) acreditaram que adquiriram maior confiança para aplicações futuras (Tabela 2).

Nas questões abertas do questionário, 17 (24,6\%) estudantes destacaram como pontos fortes dos modelos a oportunidade de treinamento das técnicas sem que houvesse sofrimento e estresse animal, e 11 (15,9\%) citaram a possibilidade de repetição do treino para aprimoramento da técnica. A obtenção do sentimento de segurança e confiança para que possam executar as técnicas em animais reais foi citada por 15 $(21,7 \%)$ estudantes. Em paralelo, vários estudos demonstraram também a contribuição dos métodos substitutivos para a formação dos estudantes, uma vez que possibilitam repetição de treino e diminuem os sentimentos de estresse e nervosismo, presentes durante aulas práticas com animais vivos, possibilitando, portanto, boa assimilação de conteúdo, além da formação ética (GREIF \& TRÉZ, 2000; DINIZ et al., 2006 TRÉZ \& NAKADA, 2008; NAYLOR, 2009; CANOVA et al., 2015; NAGEL et al., 2015; DE LIMA et al., 2018).

Archives of Veterinary Science, v.25, n.4, 28-43, 2020. 
Tabela 2 - Resposta dos estudantes $(n=69)$ sobre o treinamento de aplicação de injeção pelas vias intramuscular e subcutânea em modelos sintéticos, na aula prática de Semiologia Geral Veterinária.

\begin{tabular}{|c|c|c|c|c|c|}
\hline $\begin{array}{l}\text { Em relação aos modelos apresentados para } \\
\text { aplicação de injeções em cães: }\end{array}$ & $\mathrm{DF}^{1}$ & $D^{2}$ & $\mathbf{N}^{3}$ & $\mathrm{C}^{4}$ & $\mathrm{CF}^{5}$ \\
\hline $\begin{array}{l}\text { Você considera que o uso dos modelos } \\
\text { contribuiu para o seu aprendizado }\end{array}$ & 0 & 0 & $\begin{array}{c}1 \\
(1,4 \%)\end{array}$ & $\begin{array}{c}4 \\
(5,8 \%)\end{array}$ & $\begin{array}{c}64 \\
(92,8 \%)\end{array}$ \\
\hline $\begin{array}{l}\text { Você considera que o tempo disponibilizado } \\
\text { para treinamento nos modelos foi adequado }\end{array}$ & 0 & $\begin{array}{c}1 \\
(1,4 \%)\end{array}$ & $\begin{array}{c}6 \\
(8,7 \%)\end{array}$ & $\begin{array}{c}15 \\
(21,7 \%)\end{array}$ & $\begin{array}{c}47 \\
(68,1 \%)\end{array}$ \\
\hline $\begin{array}{l}\text { Os modelos permitiram uma melhor } \\
\text { compreensão sobre a técnica para aplicação } \\
\text { de injeção pelas vias intramuscular e } \\
\text { subcutânea }\end{array}$ & 0 & $\begin{array}{c}1 \\
(1,4 \%)\end{array}$ & $\begin{array}{c}1 \\
(1,4 \%)\end{array}$ & $\begin{array}{c}6 \\
(8,7 \%)\end{array}$ & $\begin{array}{c}61 \\
(88,4 \%)\end{array}$ \\
\hline $\begin{array}{l}\text { Você se considera mais confiante após o } \\
\text { treinamento para aplicar injeção pelas vias } \\
\text { intramuscular e/ou subcutânea no animal vivo }\end{array}$ & 0 & 0 & $\begin{array}{c}5 \\
(7,2 \%)\end{array}$ & $\begin{array}{c}14 \\
(20,3 \%)\end{array}$ & $\begin{array}{c}50 \\
(72,5 \%)\end{array}$ \\
\hline
\end{tabular}

${ }^{1} \mathrm{DF}$ : Discordo fortemente, ${ }^{2} \mathrm{D}$ : Discordo, ${ }^{3} \mathrm{~N}$ : Neutro, ${ }^{4} \mathrm{C}$ : Concordo, ${ }^{5} \mathrm{CF}$ : Concordo fortemente

Como pontos fracos, 16 (23,2\%) estudantes citaram a falta de reação fisiológica do animal e 5 (7,2\%) a dificuldade em encontrar algumas referências anatômicas. A limitação presente nos modelos, em relação à anatomia, poderia ser suprida a partir do desenvolvimento e produção de bonecos com maior fidelidade anatômica. Entretanto, esta produção acarretaria maior tempo de trabalho e custo de produção. Como essa crítica em relação à anatomia partiu de uma parcela reduzida de estudantes, a possibilidade de dificuldade individual por parte destes também poderia ser considerada.

Quando questionados em relação à necessidade da aula prática apenas com o modelo, apenas com o animal, ou com ambos, para ensino das técnicas de aplicação de injeções, 56 (81,2\%) estudantes julgaram ser necessário modelo e animal, como foi proposto durante a aula; 10 (14,5\%) julgaram ser necessário apenas modelo, 1 (1,4\%) disse preferir aula apenas com o animal e, ainda, 2 (2,9\%) estudantes não acharam necessária aula prática. Um estudo que comparou o uso de modelo e animal vivo para aprendizado de palpação retal em éguas (NAGEL, et al., 2015), demonstrou que o treinamento repetitivo em modelo se mostrou menos eficiente para o desenvolvimento de habilidades básicas do que treinamento repetitivo em animais, porém mais eficiente do que treinamento único em animal, além de que o uso do modelo aumentou a confiança dos estudantes que o utilizaram. Foi possível observar ainda, neste mesmo estudo, que o treino em simulador pareceu ter efeito mais duradouro no aprendizado dos estudantes, quando comparados com os que fizeram uso de treinos repetitivos em animais vivos. 
Os estudantes do segundo semestre puderam praticar a aplicação de injeção subcutânea em cães vivos que necessitavam de vacinação, dois meses após a aula prática com os modelos sintéticos. No segundo questionário, após a vacinação, todos os estudantes concordaram ou concordaram fortemente que o modelo auxiliou na realização da técnica de injeção no animal vivo e acreditaram que o modelo contribuiu para que se sentissem confiantes durante a aplicação da vacina (Tabela 3). Um estudo com metodologia semelhante (NIBBLETT et al., 2017) comparou o uso de modelo tridimensional de orelha canina para treinamento de otoscopia e a realização do exame em animal vivo. Todos os estudantes que treinaram no modelo concordaram ou concordaram fortemente que o mesmo se mostrou útil nos aspectos de aprendizado, conhecimento e confiança. Os autores observaram que alguns cães poderiam apresentar aversão à introdução do otoscópio e realização do exame, prejudicando, portanto, o aprendizado do estudante e, consequentemente, a diminuição da sua confiança para a realização de exames futuros. Esse comportamento reativo que eventualmente pode ser observado em alguns cães durante as aulas práticas, poderia também ser apresentado pelos animais que foram submetidos à vacinação no presente estudo. Levando em consideração que os cães submetidos às vacinas pertenciam à raça Beagle, conhecida por ser dotada de grande atividade e energia, poderiam dificultar a contenção e aplicação das injeções. Portanto, supõe-se que o treinamento prévio em modelo sintético foi útil para que os estudantes se sentissem mais preparados e confiantes para a aplicação da injeção. A segurança e confiança para executar a técnica posteriormente em animais reais também foi verificada em um estudo realizado com simuladores para ensino de técnica cirúrgica veterinária (MARTINS FILHO, 2015).

Foi observado por meio da aplicação de questionários durante o treinamento em modelo sintético e pós-treinamento com a aplicação em animais vivos, que 8 (40\%) estudantes não se sentiriam confortáveis para a realização do procedimento sem treinamento prévio no modelo. O estudo de LANGEBAEK, et al. (2012) corrobora com esse dado ao mostrar que estudantes que tiveram contato direto com animais vivos na cirurgia veterinária, demonstraram emoções negativas como o nervosismo, a ansiedade, além da falta de autoconfiança, e acreditaram que essas atrapalharam seu aprendizado e desempenho. Outro estudo, envolvendo estudantes de Ciências Biológicas, Farmácia e Medicina, demonstrou que dentre os que participaram de aulas práticas com o uso de animais vivos, 63,5\% sentiram algum tipo de desconforto (TRÉZ, 2015). Ademais, o Conselho Nacional de Controle da Experimentação Animal (CONCEA) e as Comissões de Ética no Uso de Animais (CEUAs), criados a partir da Lei Arouca (11.794/2008), atualmente indicam o uso de modelos antes da prática em animais vivos, a fim de que os estudantes possam 
obter o aperfeiçoamento de seus aprendizados, além de reduzir riscos e possíveis danos aos animais. Ainda que 12 (60\%) dos 20 estudantes que aplicaram as vacinas tenham dito que se sentiriam confortáveis ou indiferentes ao aplicar injeção no animal vivo sem treinamento prévio com modelo, vale ressaltar que todos concordaram ou concordaram fortemente que o modelo contribuiu para a aplicação de vacinas, portanto, a aula prática com modelo sintético trouxe benefício a todos que o utilizaram.

Tabela 3 - Respostas dos estudantes $(n=20)$ sobre a utilidade dos modelos, após experiência de aplicação de injeção pela via subcutânea (vacina) em cães, durante aula prática de Semiologia Geral Veterinária.

\begin{tabular}{|c|c|c|c|c|c|}
\hline $\begin{array}{l}\text { Em relação aos modelos para aplicaçãa de } \\
\text { injeção e a prática de vacinação em cães: }\end{array}$ & $\mathrm{DF}^{1}$ & $D^{2}$ & $\mathbf{N}^{3}$ & $C^{4}$ & $\mathrm{CF}^{5}$ \\
\hline $\begin{array}{l}\text { Considera que o treinamento anterior (aula } \\
\text { prática com os modelos de aplicação de } \\
\text { injeções), contribuiu para a prática da aplicação } \\
\text { da vacina }\end{array}$ & 0 & 0 & 0 & $\begin{array}{c}2 \\
(10 \%)\end{array}$ & $\begin{array}{c}18 \\
(90 \%)\end{array}$ \\
\hline $\begin{array}{l}\text { Considera que a sensação ao introduzir a agulha } \\
\text { no modelo foi semelhante ao animal }\end{array}$ & 0 & 0 & $\begin{array}{c}2 \\
(10 \%)\end{array}$ & $\begin{array}{c}10 \\
(50 \%)\end{array}$ & $\begin{array}{c}8 \\
(40 \%)\end{array}$ \\
\hline $\begin{array}{l}\text { Considera que a prega de pele do modelo foi } \\
\text { semelhante à do animal }\end{array}$ & 0 & 0 & $\begin{array}{c}2 \\
(10 \%)\end{array}$ & $\begin{array}{c}9 \\
(45 \%)\end{array}$ & $\begin{array}{c}9 \\
(45 \%)\end{array}$ \\
\hline $\begin{array}{l}\text { Considera que o treinamento no modelo } \\
\text { contribuiu para a sua confiança no momento da } \\
\text { aplicação da vacina }\end{array}$ & 0 & 0 & 0 & $\begin{array}{c}6 \\
(30 \%)\end{array}$ & $\begin{array}{c}14 \\
(70 \%)\end{array}$ \\
\hline $\begin{array}{l}\text { Você se considera mais confiante após o } \\
\text { treinamento no modelo e nos animais para } \\
\text { aplicar injeção subcutânea sozinho(a)? }\end{array}$ & 0 & 0 & 0 & $\begin{array}{c}3 \\
(15 \%)\end{array}$ & $\begin{array}{c}17 \\
(85 \%)\end{array}$ \\
\hline $\begin{array}{l}\text { Se você não tivesse tido a prática anterior com o } \\
\text { modelo de injeção subcutânea, se sentiria } \\
\text { confortável em realizar a vacinação nos animais } \\
\text { da prática de hoje? }\end{array}$ & $\begin{array}{c}2 \\
(10 \%)\end{array}$ & $\begin{array}{c}6 \\
(30 \%)\end{array}$ & $\begin{array}{c}10 \\
(50 \%)\end{array}$ & $\begin{array}{c}2 \\
(10 \%)\end{array}$ & 0 \\
\hline
\end{tabular}

${ }^{1} \mathrm{DF}$ : Discordo fortemente, ${ }^{2} \mathrm{D}$ : Discordo, ${ }^{3} \mathrm{~N}$ : Neutro, ${ }^{4} \mathrm{C}$ : Concordo, ${ }^{5} \mathrm{CF}$ : Concordo fortemente.

Como limitação neste estudo, pode ser observada a baixa durabilidade do tecido do manequim usado para injeção pela via subcutânea, uma vez que não foi fabricado com a finalidade de suportar diversos furos. Para estudos futuros, recomenda-se, portanto, a construção personalizada dos manequins, com teste prévio de tecidos para a seleção dos mais adequados. Com a construção personalizada é possível ajustar ainda o tamanho e posição anatômica desejada, sendo possível a simulação das três vias de aplicação de injeção apresentadas, em um único modelo sintético. 


\section{CONCLUSÃO}

Os modelos sintéticos de ensino propostos neste trabalho, modelo de injeção pela via intramuscular nos músculos da região femoral e lombordosal, e pela via subcutânea, proporcionaram benefícios aos estudantes, por apresentarem consistência e textura semelhantes ao animal vivo. Os modelos sintéticos foram avaliados positivamente, tanto por médicos veterinários experientes na área, quanto por estudantes. O resultado da experiência prática com a aplicação das vacinas demonstrou que o modelo de injeção subcutânea possuía características semelhantes às de um animal real nos quesitos desejados, e que o treinamento prévio foi importante no aprendizado, uma vez que todos os estudantes afirmaram que o modelo os deixou mais confiantes.

\section{NOTAS INFORMATIVAS}

O projeto foi aprovado pela CEUA sob o protocolo n. 003/2019.

\section{REFERÊNCIAS}

CANOVA, F.; SILVA, P.C.; GRASSI-KASSISSE, D.M. Alternativas para a diminuição do uso de animais na educação. Revista Neurociências, v.23, n.2, p.313-316, 2015.

CARDOSO, T.L.; LANGE, R.R. O uso de animais no ensino de odontologia veterinária: percepção dos alunos quanto a utilização de método alternativo. Archives of Veterinary Science, v.20, n.2, p.132-140, 2015.

CROW, S.E.; WALSHAW S.O. Manual de Procedimentos Clínicos em Cães, Gatos e Coelhos. $1^{\circ}$ ed. Porto Alegre: Artemed, 2000. 280p.

DE LIMA, F.T.; STURN, R.M.; RIBEIRO, A.R.B. Uso de animais no ensino da medicina veterinária: métodos substitutivos. Nucleus, v.15, n.2, p.251-264, 2018.

DINIZ, R.; DUARTE, A.L.A.; OLIVEIRA, C.A.S. et al. Animais em aulas práticas: podemos substituí-los com a mesma qualidade de ensino. Revista Brasileira de Educação Médica, v.30, n.2, p.31-41, 2006.

GREIF, S.; TRÉZ, T.A. A Verdadeira Face da Experimentação Animal: A sua saúde em perigo. Rio de Janeiro: Sociedade Educacional Fala Bicho, 2000. 81 p.

KNIGHT, A. The effectiveness of humane teaching methods in veterinary education. Alternatives to animal experimentation, v.24, n.2, p.91-109, 2007.

LANGEBAEK, R.; EIKA, B.; TANGGAARD, L. et al. Emotions in veterinary surgical students: A qualitative study. Journal of Veterinary Medical Education, v.39, n.4, p.312-321, 2012.

MAGALHÃES, M.; ORTÊNCIO FILHO, H. Alternativas ao uso de animais como recurso didático. Arquivo de Ciências Veterinárias e Zoologia da Unipar, Umuarama, v.9, n.2, p.147-154, 2006. 
MARTINS FILHO, E.F. Métodos Alternativos no Ensino da Técnica Cirúrgica Veterinária. 2015. São Paulo, 117f. Tese (Doutorado em Cirurgia Veterinária) - Programa de PósGraduação em Cirurgia Veterinária. Universidade Estadual Paulista, Faculdade de Ciências Agrárias e Veterinárias.

NAGEL, C.; ILLE, N.; AURICH, J. et al. Teaching of diagnostic skills in equine gynecology: Simulator-based training versus schooling on live horses. Theriogenology, v.84, n.7, p.1088-1095, 2015.

NAYLOR, R.A.; HOLLETT, L.A.; VALENTINE, R.J. et al. Can medical students achieve skills proficiency through simulation training? The American Journal of Surgery, v.198, n. 2, p.277-282, 2009.

NIBBLETT, B.M.D.; PEREIRA, M.M.; SITHOLE, F et al.. Design and Validation of a ThreeDimensional Printed Flexible Canine Otoscopy Teaching Model. Simulation in Healthcare, v.12, n.2, p.91-95, 2017.

SMEAK, D.D. Ethical surgery training for veterinary students. In: CHIUIA, M.; JUKES, N. Guinea Pig to Computer Mouse. 2th ed. Leicester: InterNICHE; 2006, Cap. 6, p.117-124. TAYLOR, S.M. Técnicas de injeção. In: TAYLOR, S.M. Semiotécnica de pequenos animais. $1^{\circ}$ ed. Rio de Janeiro: Elsevier, 2001. Cap.3, p.20-28.

TELLES FILHO, P.C.P.; CASSIANI, S.H.B. Administração de medicamentos: aquisição de conhecimentos e habilidades requeridas por um grupo de enfermeiros. Revista LatinoAmericana de Enfermagem, v.12, n.3, p.533-540, 2004.

TRÉZ, T.A. A caracterização do uso de animais no ensino a partir da percepção de estudantes de ciências biológicas e da saúde. História, Ciências, Saúde - Manguinhos, v.22, n.3, p.863-880, 2015.

TRÉZ, T.A.; NAKADA, J.I.L. Percepções acerca da experimentação animal como um indicador do paradigma antropocêntrico-especista entre professores e estudantes de ciências biológicas da UNIFAL-MG. Alexandria: Revista de Educação em Ciência e Tecnologia, v.1, n.3, p.3-28, 2008. 